\title{
Bilateral Central Retinal Artery Occlusion Associated with Bilateral Lymphoproliferative Infiltrative Optic Neuropathy
}

\author{
Philip J. DeSouza ${ }^{a}$ Claudia G. Hooten ${ }^{a} \quad$ Christopher M. Lack ${ }^{b}$ Vishak J. John ${ }^{a}$ \\ Timothy J. Martin ${ }^{a}$ \\ Departments of a Ophthalmology and ${ }^{\mathrm{b}}$ Radiology, Wake Forest School of Medicine, Winston-Salem, NC, USA
}

\begin{abstract}
Keywords
Central retinal artery occlusion - Optic nerve infiltration -

Burkitt lymphoma - Leukemic central nervous system

infiltration - Post-transplant lymphoproliferative disorder .

Case report
\end{abstract}

\begin{abstract}
Background: Leukemic infiltration of the optic nerve is relatively rare. While previously described in acute leukemia, the infiltration in our case represents central nervous system (CNS) metastasis of Burkitt-type lymphoma that developed as a complication of solid-organ transplantation, resulting in a bilateral infiltrative optic neuropathy with sequential, bilateral central retinal artery occlusion (CRAO) and devastating vision loss. Methods: The medical record, serial ophthalmic examination findings, clinical course, and imaging including magnetic resonance imaging (MRI), fundus photographs, and fluorescein angiography of a single patient were retrospectively reviewed. Results: MRI demonstrated multifocal cortical and leptomeningeal CNS involvement, including the left optic nerve. Serial fundus examination/photography and fluorescein angiography showed that despite urgent whole-brain irradiation and systemic chemotherapy, CNS disease progressed to bilateral optic nerve infiltration and
\end{abstract}

\section{KARGER}

(c) 2017 S. Karger AG, Basel

E-Mail karger@karger.com

www.karger.com/oop
CRAO with no light perception vision in both eyes. Conclusion: CRAO can occur as a devastating and irreversible complication of lymphoproliferative optic nerve infiltration.

(C) 2017 S. Karger AG, Base

\section{Introduction}

Post-transplant lymphoproliferative disorder (PTLD) classically refers to the uncontrolled, neoplastic proliferation of plasmacytic or lymphoid cells as a well-recognized and potentially life-threatening complication of both solid-organ transplant (SOT) and hematopoietic stem cell transplant $[1,2]$. PTLD can be classified as early-onset $(<1$ year) or late-onset ( $>1$ year) after transplant that can manifest as a highly invasive, malignant lymphoma [3-5]. In SOT recipients, the prolonged immunosuppression necessary to prevent allograft rejection produces deficits in cytotoxic T lymphocytes, which partly function in the downregulation of B-cell proliferation [6]. The resultant unchecked growth can lead to B-cell tumor formation. Epstein-Barr virus (EBV) infection disrupts the apoptotic pathway and upregulates survival signals of B cells; it is an independent risk factor for PTLD development that is potentiated by immunosuppression [7]. Additionally, the 
Fig. 1. Right (a) and left (b) fundus photograph on initial evaluation show bilateral blurred disc margins and hyperemic vessels. The left optic disc and macula appear white with arterial "boxcarring" and scattered hemorrhages. Right (a, middle) and left (b, middle) fundus photographs 5 days later show a fluffy, white appearance over the optic discs, consistent with prelaminar infiltrate, along with hemorrhage, particularly on the nasal side of the left optic disc. Bilateral whitening of the macula is present, and the vessels are tortuous and dilated. Right (a, bottom) and left (b, bottom) photographs 13 days after initial evaluation show progression of the infiltrates, with notably increased hemorrhage surrounding the left optic disc and retina.
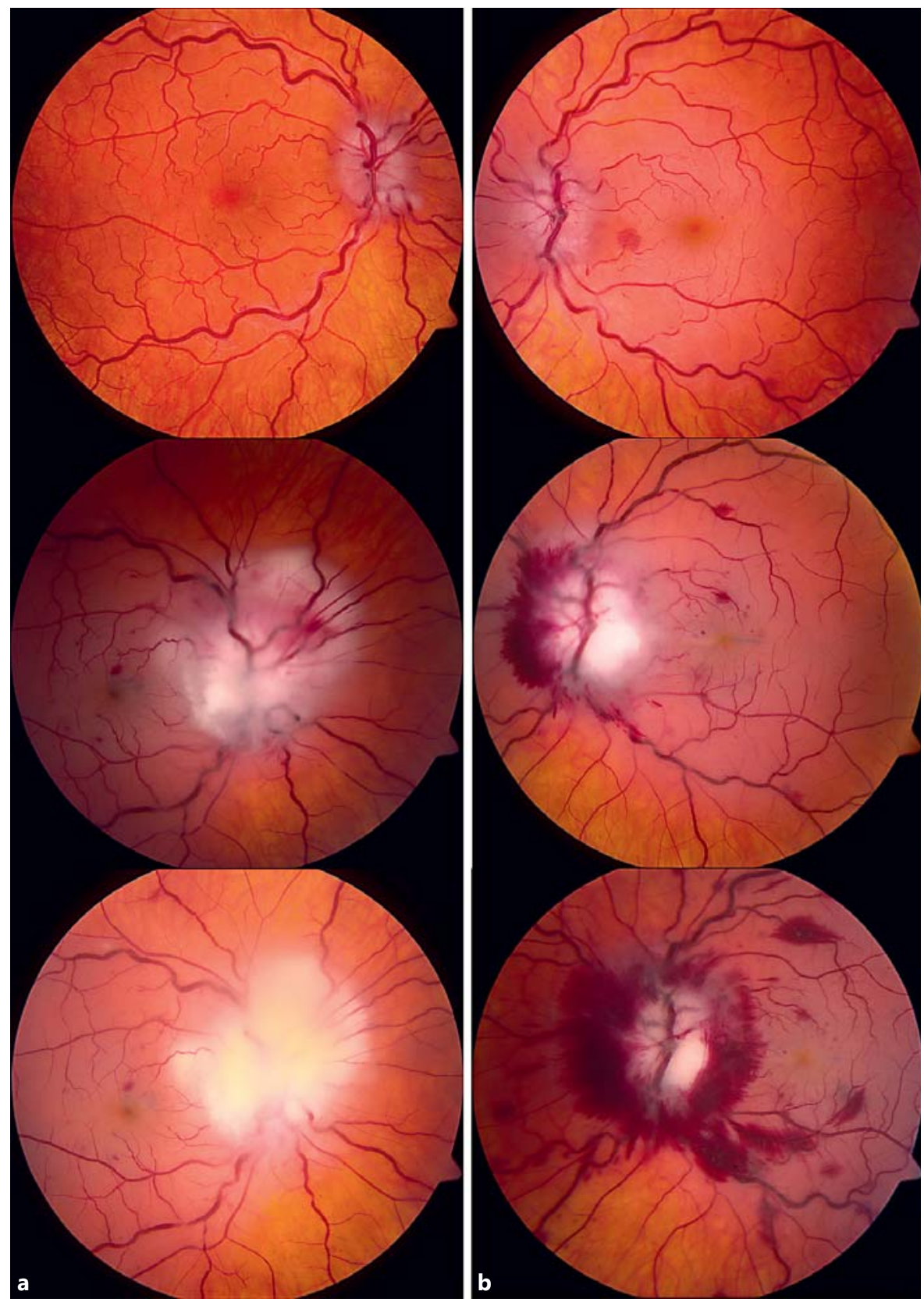

quantity of lymphoid tissue in the transplanted organ, intensity of immunosuppression, and human leukocyte antigen disparity all confer risk for PTLD development [6, 8]. In SOT, the incidence of PTLD ranges from $1-20 \%$, with the lowest risk in renal transplant recipients (1.2$9 \%)[2,6]$.

The lifetime risk for central nervous system (CNS) PTLD is 1-2\% for renal transplant patients [9]. Secondary CNS lymphoma refers to CNS localization of the lym- phoma, often within the leptomeningeal compartment, with a concomitant systemic lymphoma [10]. Ocular manifestations of CNS lymphoma or leukemized metastases include direct invasion to the orbit, eyelid, uveal tract, and retina [11]. However, direct optic nerve infiltration remains rare [11-14]. Infiltration of the optic nerve can cause vascular occlusion or compression necrosis to occur, which can lead to severe vision loss [14]. Lymphoproliferative optic nerve involvement is an oncologic 
emergency, and treatment generally involves prompt irradiation of the orbits and CNS with systemic and/or intrathecal chemotherapy $[15,16]$.

We describe a 17 -year-old male who experienced vision loss in the setting of late-onset PTLD with secondary CNS lymphoma and optic nerve involvement, demonstrated on magnetic resonance imaging (MRI). Serial ophthalmoscopy showed progressive signs of sequential bilateral central retinal artery occlusion (CRAO). Fluorescein angiography (FA) showed complete absence of dye filling the retinal arterial circulation in both eyes.

\section{Case Report}

A 17-year-old male was urgently referred to the ophthalmology clinic from the pediatric oncology clinic for headache, photophobia, and 6-h duration of complete, persistent left eye vision loss. The headache began 3 days before and coincided with a transient 10 -s loss of vision in the left eye. The patient had a history of renal transplant from an unrelated, living donor 4 years prior to presentation, maintained on tacrolimus and mycophenolic acid, due to end-stage-renal disease from nephronophthisis type 1 without ophthalmic manifestations. He was diagnosed with PTLD 4 months prior to ophthalmology evaluation after abdominal ultrasound, MR enterography, and positron emission tomographycomputed tomography (CT) showed extensive bowel lymphadenopathy and bone marrow involvement. Tissue biopsy and peritoneal fluid cytology confirmed EBV-negative, high-grade B-cell non-Hodgkin lymphoma with a t $8 ; 14)$ MYC/IGH fusion, consistent with Burkitt-type lymphoma; a peripheral blood smear reflected extensive bone marrow involvement. The patient was treated with systemic chemotherapy, with the addition of twice weekly intrathecal methotrexate and cytarabine 6 weeks before presenting to the ophthalmology clinic due to cerebrospinal fluid studies consistent with leukemic spread. CT without contrast of the brain performed at that time showed no acute intracranial abnormality.

On ophthalmic examination, visual acuity was $20 / 30$ in the right eye and no light perception (NLP) vision in the left eye, with an amaurotic left afferent pupillary defect. Intraocular pressure, extraocular motility, and the anterior segments were normal. Fundus examination showed bilateral blurred disc margins and hyperemic vessels. The left optic disc and macula appeared white with arterial "boxcarring" and scattered hemorrhages (Fig. 1b). There was concern for lymphoproliferative optic nerve infiltration with associated central artery occlusion; ophthalmic artery occlusion and papilledema from increased intracranial pressure were also considered in the differential diagnosis. The patient was sent for urgent MRI of the brain and orbits, which demonstrated findings concerning for multifocal cortical and leptomeningeal CNS involvement of PTLD in the anteroinferior temporal lobes, parafalcine parietooccipital cortex, pineal gland, bilateral cerebellar hemispheres, and left optic nerve (Fig. 2, 3). MRI of the spine demonstrated multifocal osseous involvement of PTLD. The patient was admitted for urgent whole-brain irradiation, 900-cGY over 12 fractions, twice daily, with systemic chemotherapy of cyclophosphamide and vincristine.

Bilateral CRAO from PTLD Optic Nerve Infiltration

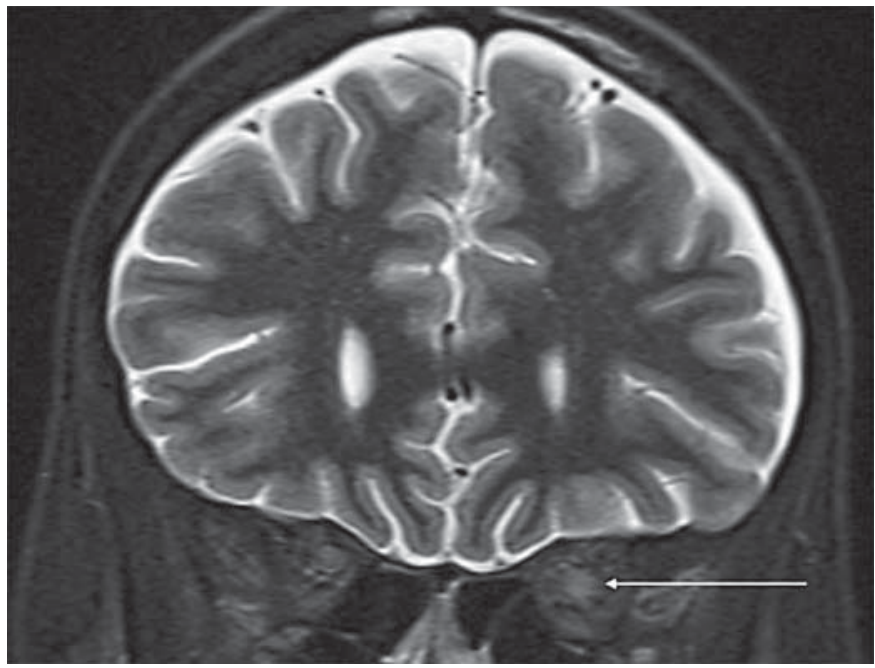

Fig. 2. Coronal short $\mathrm{T} 1$ inversion recovery magnetic resonance image showing an increased signal in the left optic nerve, consistent with optic nerve edema.

Two days later, the patient awoke with NLP vision in the right eye, pupils unreactive to light bilaterally, and increased optic disc edema in the right eye. Five days after initial ophthalmology evaluation, the patient demonstrated significant bilateral elevation of the optic discs with a pale, white, fluffy appearance and completely obscured disc margins, all consistent with lymphoproliferative infiltration of the optic nerves (Fig. 1a, b, middle). The left optic disc had a large nasal hemorrhage, and there was an increased number of left macular hemorrhages. The patient was seen again in our clinic 13 days after initial ophthalmology evaluation, with fundus examination notable for progression of right optic nerve pallor and left retinal and optic disc hemorrhage (Fig. 1a, b, bottom). FA was performed, which showed complete lack of fluorescein in the retinal vasculature throughout the entire transit cycle (Fig. 4). The patient and his family were counseled on his poor visual prognosis, and information on appropriate resources were provided. Oncologic staging was performed with flurodeoxyglucose positron emission tomography-CT, and the patient currently continues treatment with pediatric oncology.

\section{Discussion}

Our patient developed sequential, bilateral, CRAO due to lymphoproliferative infiltration of the optic nerves. While there have been case reports of leukemic infiltration of the optic nerve leading to optic disc swelling and vascular occlusion $[15,17]$, we are aware of only 1 other report in which infiltration was associated with sequential, bilateral CRAO [11]. Lin et al. [11] described a 6-year-old male with acute lymphoblastic leukemia who developed CNS relapse while on continuous che-

Ocul Oncol Pathol 2017;3:229-234 

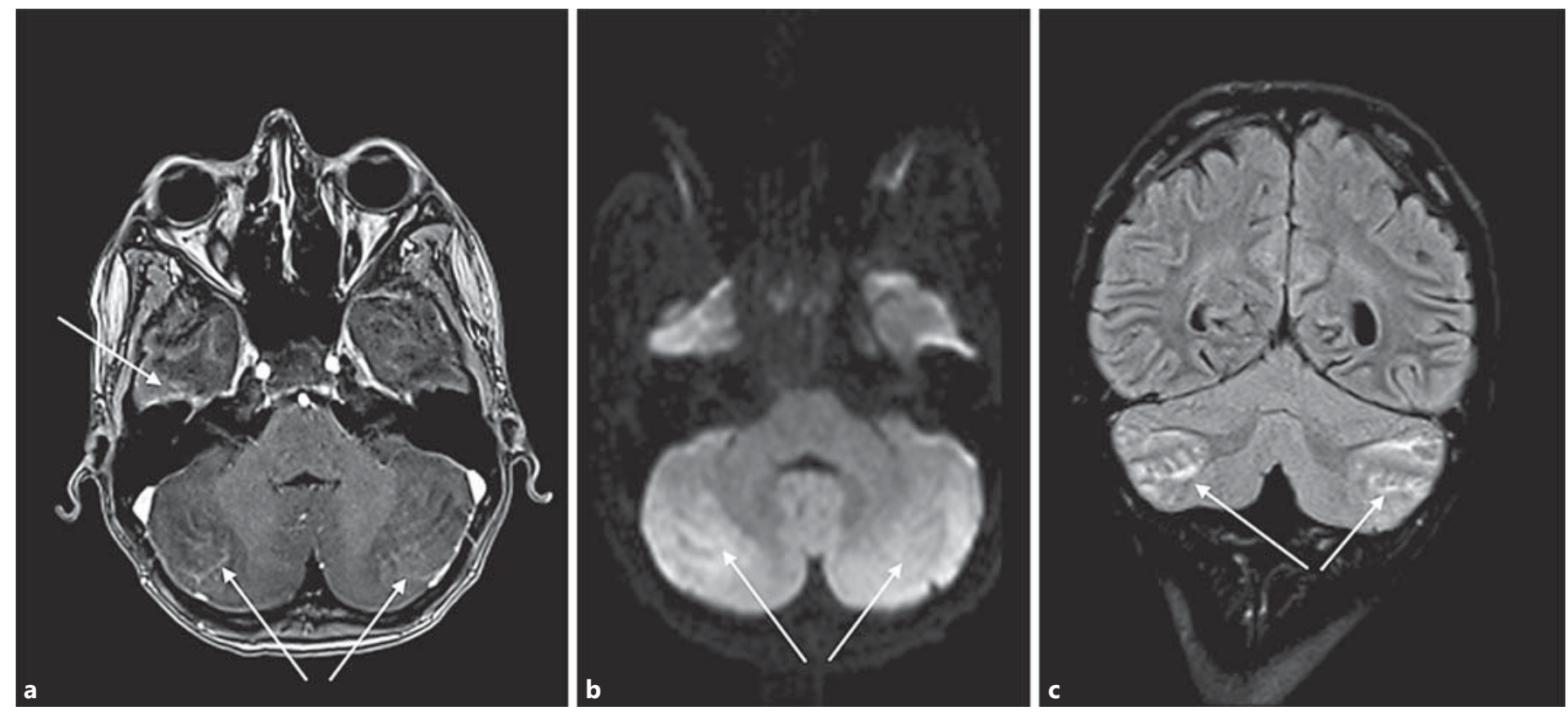

Fig. 3. a Axial T1 magnetic resonance imaging volumetric interpolated breath-hold examination showing curvilinear enhancement around the folia of the cerebellum bilaterally and along the anterior aspect of the right temporal lobe consistent with a leptomeningeal process. $\mathbf{b}$ Axial diffusion-weighted imaging showing a hyperintense signal in the area of enhancement in the bilateral cerebellar hemispheres, suggesting hypercellular material. c Coronal T2-weighted fluid attenuated inversion recovery imaging showing a hyperintense signal filling the cerebrospinal fluid spaces around the folia of the cerebellum, suggesting proteinaceous material. Overall, the findings suggest leptomeningeal involvement of the patient's known post-transplant lymphoproliferative disorder. The hyperintense signal in the anteroinferior temporal lobes, parafalcine parietooccipital cortex, and pineal gland is not shown in the figure. motherapy; despite systemic re-induction and intrathecal chemotherapy with CNS irradiation, he developed CRAO in both eyes and retained NLP vision. Our patient similarly developed optic nerve infiltration while on systemic and intrathecal chemotherapy, and CNS irradiation did not halt progression to the right optic nerve.

While native Burkitt lymphoma [18] has been associated with orbital pathology, our case represents the first PTLD Burkitt-type lymphoma associated with optic nerve infiltration and bilateral CRAO. As in the case of Lin et al. [11], optic nerve infiltration is classically associated with pediatric acute leukemia, particularly acute lymphocytic leukemia $[13,19]$, and has not been previously described with PTLD. Orbital PTLD [20] and EBVpositive chorioretinal PTLD [21] represent the only other documented PTLD ophthalmic pathologies.

Rosenthal [19] describes 2 distinct clinical patterns of optic nerve head infiltration: primary invasion of the optic nerve head (prelaminar) and primary invasion posterior to the nerve head (retrolaminar). Prelaminar infil- tration is associated with a fluffy infiltrate superficial to the lamina cribosa with edema and varying amounts of hemorrhage and visual acuity altered minimally if there is no macular involvement. Retrolaminar infiltration, posterior to the lamina cribosa, is associated with a profound decrease in visual acuity, disc elevation, and edema. Our patient initially presented with retrolaminar infiltration that progressed to a prelaminar infiltration with correlated changes in optic disc appearance and visual acuity.

While we considered papilledema in our initial differential diagnosis, it is critical to differentiate it from optic nerve infiltration, which requires urgent treatment with irradiation and/or chemotherapy to attempt to restore vision. It is likely that papilledema due to meningeal infiltration occurs simultaneously with optic nerve infiltration, so a high degree of suspicion for optic nerve infiltration must be maintained [19]. In our case, MRI showed optic nerve involvement, but lumbar puncture can also allow for differentiation so that proper, timely treatment for either condition can be executed. 

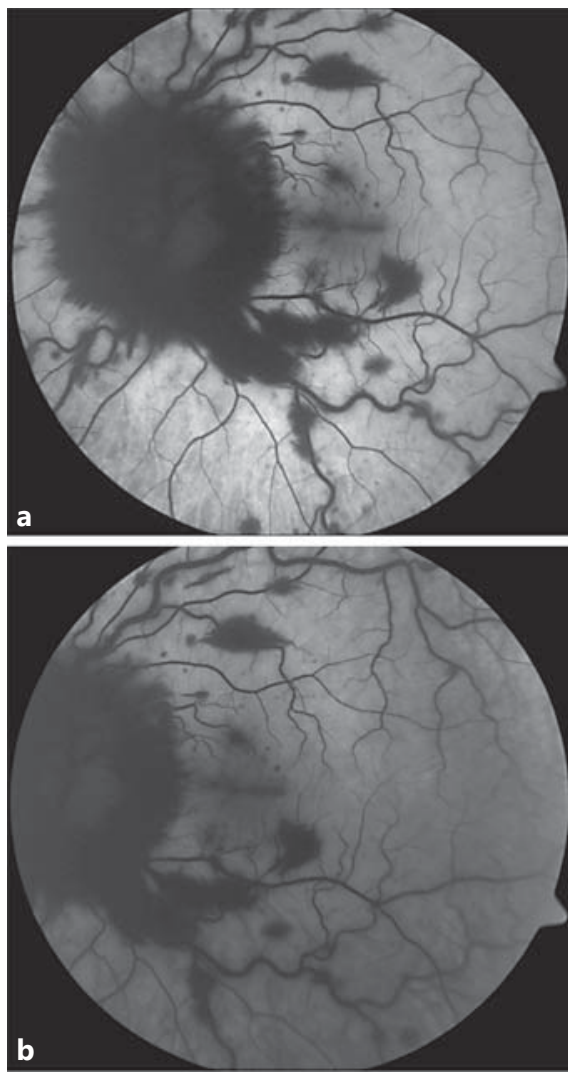

Fig. 4. Fluorescein angiogram of the left eye demonstrating early (a) and late (b) hypofluorescence in the retinal vasculature, consistent with central retinal artery occlusion.

Urgent CNS irradiation was initiated in our patient; systemic chemotherapy continued to be administered but intrathecal therapy was paused due to concern for CNS toxicity. Though CNS irradiation has been shown to restore visual acuity to baseline or near-baseline in leukemic optic neuropathy $[17,22]$, this was unfortunately not the case in our patient. As our patient had initially developed CRAO in the left eye, visual prognosis of the left eye was already guarded regardless of CNS irradiation; however, there was hope of halting the progression of right optic nerve infiltration and subsequent CRAO development. Within $48 \mathrm{~h}$ of initiating CNS irradiation, the patient developed a right CRAO with NLP vision. Serial fundus examination demonstrated the rapid progression of retrolaminar to prelaminar infiltration with macular whitening and foveal "cherry-red spots" (Fig. 1). FA confirmed a complete lack of retinal vascular circulation (Fig. 4). This finding occurred despite an almost complete course of CNS irradiation, indicating irreversible retinal ischemia due to occlusion of the retinal vasculature by optic nerve infiltration. Though there remains no consensus on the optimal management of optic nerve infiltration, timely CNS irradiation is generally considered the first-line treatment of choice. The optic nerve is considered a "pharmacologic sanctuary" from intrathecal or systemic cytotoxic drugs alone [13], but irradiation is thought to synergistically allow the drugs to reach and act on the neoplastic cells within the optic canal.

EBV-negative PTLD Burkitt-type lymphoma has a very poor prognosis and only minimally responds to even highly-aggressive chemotherapy protocols [4], which offers some explanation to why the patient developed optic nerve infiltration despite ongoing systemic and intrathecal chemotherapy. Rituximab, a B-cell antibody, has been used with success to treat PTLD, but our patient had previously failed a chemotherapy regimen of rituximab, cyclophosphamide, and prednisone, further underscoring the severity of his disease. This aggressiveness has been well-reported [23]. In our patient, a normal CT of the brain performed 6 weeks before initial optic nerve involvement suggests a rather rapid progression of the patient's CNS disease, although CT has a low sensitivity for detection of leptomeningeal disease compared with contrast-enhanced MRI.

We present this case to illustrate the unique presentation of Burkitt-type PTLD infiltration of the optic nerve with sequential, bilateral CRAO. Optic nerve infiltration should be considered not only in patients with acute lymphocytic or lymphoblastic leukemias, but also in patients with PTLD. Vascular occlusion represents a dire complication of leukemic optic nerve infiltration, and urgent irradiation and chemotherapy should be administered to prevent bilateral disease.

\section{Statement of Ethics}

This study was conducted in compliance with the rules and regulations of the United States of America's Health Insurance Portability and Accountability Act of 1996 as well as in adherence to the Declaration of Helsinki and all other relevant federal and state laws. The nonidentifiable data of this study is considered exempt research by the University's Institutional Review Board.

\section{Disclosure Statement}

The authors report no conflicts of interest. 


\section{References}

1 Savani BN, Mohty M: Clinical Guide to Transplantation in Lymphoma. Chichester, John Wiley \& Sons, 2015.

2 Taylor AL, Marcus R, Bradley JA: Post-transplant lymphoproliferative disorders (PTLD) after solid organ transplantation. Crit Rev Oncol Hematol 2005;56:155-167.

3 Quinlan SC, Pfeiffer RM, Morton LM, Engels EA: Risk factors for early-onset and late-onset post-transplant lymphoproliferative disorder in kidney recipients in the United States. Am J Hematol 2011;86:206-209.

4 Naik S, Tayapongsak K, Robbins K, Manavi CK, Pettenati MJ, Grier DD: Burkitt's lymphoma presenting as late-onset posttransplant lymphoproliferative disorder following kidney and pancreas transplantation: case report and review of the literature. Case Rep Oncol 2013;6:6-14.

5 Loren AW, Porter DL, Stadtmauer EA, Tsai DE: Post-transplant lymphoproliferative disorder: a review. Bone Marrow Transplant 2003;31:145-155.

6 Lim WH, Russ GR, Coates PT: Review of Epstein-Barr virus and post-transplant lymphoproliferative disorder post-solid organ transplantation. Nephrology 2006;11:355-366.

7 Ghigna M-R, Reineke T, Rincé P, Schüffler P, El Mchichi B, Fabre M, Jacquemin E, Durrbach A, Samuel D, Joab I, Guettier C, Lucioni M, Paulli M, Tinguely M, Raphael M: Epstein-Barr virus infection and altered control of apoptotic pathways in posttransplant lymphoproliferative disorders. Pathobiol J Immunopathol Mol Cell Biol 2013;80: 53-59.

8 Uhlin M, Wikell H, Sundin M, Blennow O, Maeurer M, Ringden O, Winiarski J, Ljungman P, Remberger M, Mattsson J: Risk factors for Epstein-Barr virus-related post-transplant lymphoproliferative disease after allogeneic hematopoietic stem cell transplantation. Haematologica 2014;99:346-352.

9 Schabet M: Epidemiology of primary CNS lymphoma. J. Neurooncol 1999;43:199-201.

10 Rubenstein JL, Gupta NK, Mannis GN, LaMarre AK, Treseler P: How I treat CNS lymphomas. Blood 2013;122:2318-2330.

11 Lin F-C, Chen J-T, Horng C-T: Bilateral central retinal artery occlusion associated with leukemic optic neuropathy. Can J Ophthalmol 2007;42:759-760.

12 Brown GC, Shields JA: Tumors of the optic nerve head. Surv Ophthalmol 1985;29:239264.

13 Nikaido H, Mishima H, Ono H, Choshi K, Dohy H: Leukemic involvement of the optic nerve. Am J Ophthalmol 1988;105:294-298.

14 Wallace RT: Leukemic Infiltration of the Optic Nerve. Arch Ophthalmol 1991;109:1027.

15 Shah P, Yohendran J, Lowe D, McCluskey P: Devastating bilateral optic nerve leukaemic infiltration: letter to the editor. Clin Experiment Ophthalmol 2012;40:e114-e115.
16 Puvanachandra N, Goddard K, Lyons CJ: Dramatic visual recovery after prompt radiotherapy and chemotherapy for leukaemic infiltration of the optic nerve in a child. Eye Lond Engl 2010;24:927-928.

17 Lin YC, Wang AG, Yen MY, Hsu WM: Leukaemic infiltration of the optic nerve as the initial manifestation of leukaemic relapse. Eye 2004; 18:546-550.

18 Lopes AJ: Burkitt Lymphoma with initial clinical presentation due to infiltration of the central nervous system and eye orbits. Am J Case Rep 2014;15:404-410.

19 Rosenthal AR: Ocular manifestations of leukemia. Ophthalmology 1983;90:899-905.

20 Douglas RS, Goldstein SM, Katowitz JA, Gausas RE, Ibarra MS, Tsai D, Sharma A, Nichols C: Orbital presentation of posttransplantation lymphoproliferative disorder: a small case series. Ophthalmology 2002;109:2351-2355.

21 Demols PF, Cochaux PM, Velu T, CaspersVelu L: Chorioretinal post-transplant lymphoproliferative disorder induced by the Epstein-Barr virus. Br J Ophthalmol 2001;85: 93-95.

22 Rosenthal AR, Egbert PR, Wilbur JR, Probert JC: Leukemic involvement of the optic nerve. Trans Pac Coast Otoophthalmol Soc Annu Meet 1974;55:137-158.

23 Dierickx D, Tousseyn T, Gheysens O: How I treat posttransplant lymphoproliferative disorders. Blood 2015;126:2274-2283. 